\title{
Die Vielfalt der Zeiten
}

Die Zeit ist Anlass vielfältiger Probleme - und das nicht nur bei Menschen mit psychischen Problemen. In vier Thesen erörtert Daniel Hell die Modalitäten der Zeit, analysiert, wodurch das Zeiterleben beeinflusst wird, wie es sich auf unser Leben auswirkt - und wie der rinnenden Zeit Haltepunkte entgegengesetzt werden können, um sich nicht in der Hast zu verlieren.

Thomas Mann fasst im «Zauberberg» das jahrtausendealte Ringen um die Zeit kurz und bündig zusammen: «Die Zeit - ein Geheimnis, wesenlos und allmächtig». Wir wissen zwar nicht, was Zeit ist, aber wir wissen um unsere Probleme mit der Zeit - gerade im ärztlichen Alltag. Auch unsere Patienten leiden vielfach an Zeitproblemen. So haben Stresserkrankungen mit drängender Zeit zu tun. Darüber hinaus gehen manche psychische Erkrankungen mit einem veränderten Zeiterleben einher, das eigene Probleme schafft. Es dürfte sich deshalb lohnen, sich grund-

\section{Menschen mit bipolaren affektiven Störungen kennen sowohl den Stillstand der Zeit wie das Rasen der Zeit.}

Korrespondenz:

Prof. em. Dr. med. Daniel Hell Privatklinik Hohenegg AG

Postfach 555

CH-8706 Meilen

Tel. 0449251212

daniel.hell[at]hohenegg.ch sätzlicher mit den Modalitäten der Zeit zu befassen und dabei den Einfluss kultureller Zeitverständnisse mit zu berücksichtigen. Mein Beitrag gliedert sich in vier Punkte oder Thesen. Die erste These behandelt die Vielfalt der Zeiten:

\section{Zeit wird nicht gleichartig erlebt, weder kulturell noch individuell.}

Wenn wir kulturgeschichtlich eine vormoderne, eine moderne und eine postmoderne Zeit unterscheiden, verweist dies auch darauf, dass sich das Zeiterleben der Menschen epochal verändert hat. Vor der Erfindung der mechanischen Uhr, also in der Vormoderne, war das Zeiterleben naturgebunden. Nach und nach wurde es in der Moderne immer stärker mit der Uhrzeit verknüpft. Heute ist es postmodern zunehmend von den elektronischen Medien abhängig. Nicht umsonst bezeichnet man die Moderne auch als «Neuzeit», also als neue (mechanisierte) Zeit und charakterisiert die Postmoderne als nachkommende (elektronische, vernetzte) Zeit. Man muss nicht wie Karl Heinz Geissler, dem ich diese chronosoziologischen Ansätze verdanke, der Auffassung sein, dass die Schule in der Aufklärungszeit hauptsächlich dazu eingerichtet wurde, um die Menschen der Uhr zu unterwerfen und um sie schliesslich an den Arbeitstakt und an die Stempeluhr zu gewöhnen. Doch die Erfindung der Uhr hat die Menschen in einer bestimmten Weise tatsächlich aus dem naturgegebenen Rhythmus geworfen. Das geschah nicht von heute auf morgen und war auch durch andere technische Erfindungen (wie Elektrizität und Motorenkraft) mitbedingt. Aber der gnadenlose Takt der Mechanik, der keine Abweichung kennt, ersetzte mehr und mehr den Rhythmus der Natur, der weniger regelmässig ist und mehr einem Auf und Ab gleichkommt.

Die Erfindung der Uhr trug mit dazu bei, dass sich die Lebensführung in den letzten Jahrhunderten zunehmend beschleunigte. Heute scheint eine weitere Beschleunigung der linearen Zeit nur noch begrenzt möglich. Doch erlauben uns in der Postmoderne neue Technologien (wie PC oder Smartphones) nicht nur ein schnelles Nacheinander, sondern auch ein vernetztes Miteinander, ein «Multitasking». Diese technische Revolution wird unser Zeiterleben weiter verändern.

Neben den wichtigen kulturellen Einflüssen wird Zeit aber auch individuell unterschiedlich erlebt. Am eindrücklichsten - wie unter einer Lupe - illustrieren dies psychisch kranke Menschen, vor allem Menschen mit bipolaren affektiven Störungen. Diese episodische Erkrankungsform kennt sowohl den Stillstand der Zeit wie das Rasen der Zeit. So erlebt ein bipolarer Patient in schwerer Depression, dass seine innere Uhr wie stillsteht. «In allem, was ich tue, komme ich nicht voran, dabei habe ich so viel zu tun. Aber ich bin wie gelähmt. Ich bleibe hinter meinen Pflichten zurück. Ich stehle Zeit.» Der Fluss der Zeit ist wie angehalten.

Anders im manischen Zustand. Hier empfindet der bipolare Patient, dass nicht er, sondern seine Umwelt langsam - im Zeitlupentempo - agiert. Seine Zeit hingegen rast. Er erlebt seine Tage wie mit einem Zeitraffer gedrängt und viel zu kurz, als dass alles umzusetzen wäre, was ihm in Gedanken und Plänen durch den Kopf geht. 
Der Gegensatz von depressivem und manischem Zeiterleben könnte nicht grösser sein. Aber die extreme Polarität spiegelt nur wider, was auch Gesunde in gemässigter Form kennen. So schleicht etwa die Zeit, wenn man auf einen verspäteten Zug warten muss. Es packt einen dann Langeweile. Andererseits vergessen wir die Zeit im Sinnesrausch eines packenden Films. Die Zeit geht dann wie im Nu vorbei.

\section{Ohne Zweifel braucht es das Verweilen im Augenblick,} um einen Weg zu öffnen, der über das Vorgegebene

\section{hinausführt.}

Angesichts dieser Zeitvielfalt in biographischer wie kultureller Hinsicht erscheint eine erzwungene Reduktion des Erlebens auf eine einzige Zeitdimension, sei es die Vergangenheit oder Zukunft, problematisch. Darauf basiert die zweite These:

\section{Unfrei und belastet ist,}

wer von der Vergangenheit beherrscht wird.

Als Beispiel dieser Problematik kann die melancholische Form der monopolaren Depression dienen.

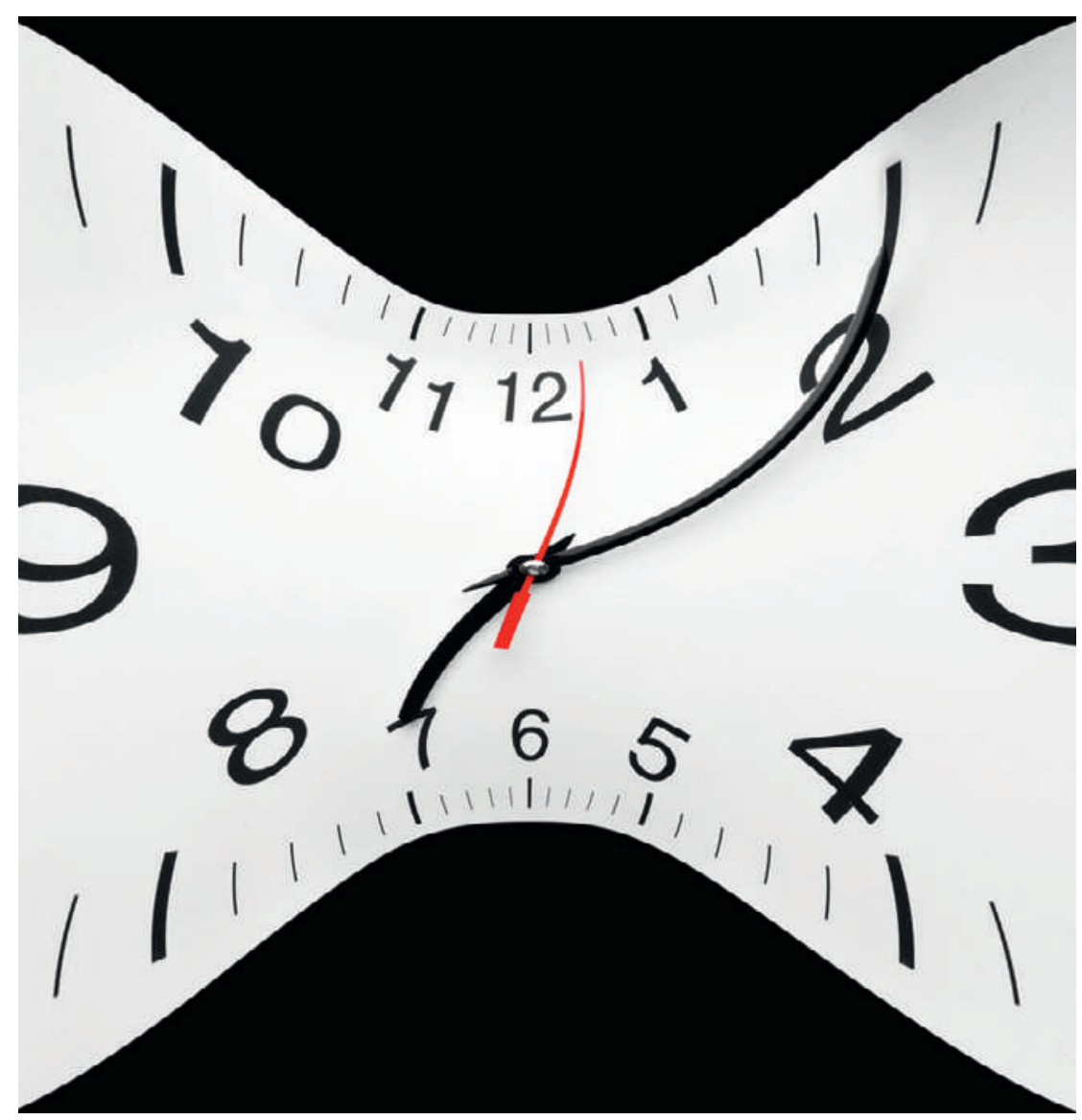

Mal rast sie, mal scheint sie stillzustehen - Zeit wird individuell und situativ sehr unterschiedlich erlebt.
Hier dehnt sich die Zeit nicht nur, sondern verharrt ebenso in der Vergangenheit. Der Heidelberger Psychiater Tellenbach hat die Disposition zu dieser Erlebensweise «Typus Melancholicus» genannt, weil sie durch eine sogenannte Remanenz, also einem Haften am Vergangenen, charakterisiert ist. Die Ordentlichkeit, zu der diese Menschen neigen, schliesst sie gleichsam von der Zukunft ab. Es darf nichts offen bleiben, alles muss seinen bestimmten und gewohnten Gang nehmen. Die Vergangenheit bekommt im Leben dieser Menschen mithin ein so grosses Gewicht, dass spontane Entscheide kaum möglich sind. Die Vergangenheit saugt gleichsam die Zukunft auf.

Es scheint nun nicht zufällig, dass depressive Menschen dieses melancholischen Typus besonders stark unter Skrupeln leiden und sich Schuldvorwürfe machen. Denn gerade die Ordentlichkeit und Gewissenhaftigkeit lässt sie am Vergangenen festhalten und macht es ihnen schwer, vom Vergangenen abzusehen und, was geschehen ist, loszulassen. Im Lichte dieser besonderen depressiven Zuspitzung wird deutlich, dass die Herrschaft der Vergangenheit unfrei macht.

Nun ist niemand, der ein Gewissen hat und sich schämen kann, davor gefeit, mit belastenden Erinnerungen konfrontiert zu werden, die auch Selbstvorwürfe wecken. Aber die Herrschaft der Vergangenheit kann vermieden werden, wenn es gelingt, die dimensionale Polarität von Vergangenheit, Gegenwart und Zukunft aufrechtzuerhalten und sich in keiner Zeitdimension zu verlieren, also auch nicht in der Zukunft.

Daran schliesst die dritte These an:

\section{Unter Anspannung leidet,} wer von der Zukunft beherrscht wird.

Das klinische Beispiel für Menschen, die in der Gewalt der Zukunft stehen, sind Angst- und Zwangskranke. Angst und Furcht sind zukunftsbezogen. Wo sie überhand nehmen, schliesst die Zukunft die Gegenwart und Vergangenheit immer mehr aus. Auch Zwangsgedanken sind in der Regel Befürchtungen - z. B. sich zu beschmutzen -, die mit Zwangshandlungen, z. B. mit einem Waschzwang, abgewehrt werden. Aber Ängste und ihre Abwehr gehören bis zu einem bestimmten Ausmass zur «condition humaine» und sind von unserem Alltagserleben nicht völlig wegzudenken. Sie hängen mit der «exzentrischen Positionalität» (Plessner) des Menschen zusammen, also der Fähigkeit, uns von aussen wahrzunehmen und uns z.B. vorzustellen, was auf uns zukommt. Vielleicht ist es sogar so, (wie die Daseinsanalytikerin Alice Holzhey annimmt), dass Menschen ihre existentielle Angst, auch ihr Wissen um den Tod, einzugrenzen versuchen, indem sie sie phobisch an bestimmte lokalisierbare Befürchtungen binden. Sie werden nach dieser Konzeption vor allem dann von Phobien geplagt, wenn sie für die existentielle Angst besonders sensibel sind.

Die vierte These fasst die ersten drei zusammen und zieht daraus praktische Schlussfolgerungen: 
Am besten scheinen Menschen mit der Zeit zurechtzukommen, wenn sie die Vielfalt der Zeiten akzeptieren und sie synthetisierend und rhythmisierend miteinander verbinden.

Der Zeitforscher Karl Heinz Geissler, der ohne Uhr lebt, schreibt in seinem lesenswerten Buch «Alles hat seine Zeit, nur ich habe keine»: «Das, was wir «Leben nennen, zeichnet sich durch ein Konglomerat, ein abwechslungsreiches Kunterbunt aus Zeitformen und Zeitqualitäten, Zeitzuständen und Zeiterfahrungen aus. Das Leben ist multitemporal und die Welt ist es ebenso. (...) Das Leben kann nur dort frei von Zeitnöten, Zeitkonflikten und Zeitproblemen gelebt werden, wo sich die Welt reich an Zeitqualitäten zeigt. »

Nach dieser Einsicht zu leben, bedeutet aber, weder der mechanischen Uhr noch den elektroni- nisch-wissenschaftlichen Zivilisation, dass ein kurzes kontemplatives Unterbrechen seinen Aktivitätsrausch schwerlich zum Abklingen bringen kann.

Es braucht wohl eine tiefere Auseinandersetzung mit dem, was ist (und dem, dass etwas ist und nicht nichts ist), um zum «Sein» zu finden. Ohne Zweifel braucht es aber das Verweilen im Augenblick, um einen Weg zu öffnen, der über das Vorgegebene hinausführt. Diesen Weg kann man aber nicht schnell durch mentale Techniken womöglich beschleunigt hinter sich bringen. Er gehört als Weg bereits zum Ziel. Das lehrt auch der spirituelle Erfahrungsschatz aller Hochreligionen.

Eine von alters her bekannte Hilfe, diesen Weg $\mathrm{zu}$ beschreiten, ist die Rhythmisierung des Lebens. Damit ist ein Wechsel von Tätigkeit und Musse, ein

\section{Es liegt hauptsächlich am Einzelnen, aus ständiger Erreichbarkeit und ruheloser Tätigkeit auszubrechen, um aktive Zeiten mit Zeiten der Ruhe zu verbinden.}

schen Medien die Herrschaft über uns zu überlassen. Gerade die Dimensionalität des Zeiterlebens, dass wir nämlich von der Gegenwart aus auf Vergangenheit und Zukunft zugreifen können, kann der linearen Zeit der technisch-wissenschaftlichen Epoche, die nur Zeitatome, aber keinen Augenblick kennt, bereits ein Schnippchen schlagen. Das hatte wohl Martin Heidegger im Sinn, wenn er sich gegen das «zerstreute Unverweilen» wandte, also gegen das atemlose Hasten in der Moderne. Er schreibt (in den «Grundbegriffen der Metaphysik»): «Warum haben wir keine Zeit? Inwiefern wollen wir keine Zeit verlieren? Weil wir sie brauchen und verwenden wollen. Wofür? Für unsere alltäglichen Beschäftigungen, deren Sklaven wir längst geworden sind. (...) Am Ende ist dieses Keine-Zeit-haben eine grössere Verlorenheit des Selbst als jenes Sich-Zeit-lassende-Zeitverschwenden.»

Heidegger beschwört das «Wesentliche im Dasein», das nur im Verweilen gefunden werden kann, während die Eile zur «Uneigentlichkeit» oder zur «Seinsvergessenheit» führe.

Es bleibt aber die Frage, ob Kontemplation allein ohne entsprechende spirituelle Kultur - der stets drohenden Herrschaft der Zeit Herr werden kann. Der moderne Mensch hat so viele Placebos der Ablenkung zur Verfügung und ist so stark geprägt von der tech-
Vor und Zurück in vita activa und vita contemplativa, gemeint. Der physikalisch rinnenden Zeit sollen feste Haltepunkte entgegengesetzt werden, um sich nicht in Hast oder Zerstreuung zu verlieren.

Eine solche Rhythmisierung ist heute zur individuellen Herausforderung geworden, weil das moderne Leben an zyklischen Taktgebern arm geworden ist und kulturell vorgegebene Rhythmen weitgehend fehlen. Die Technisierung hat die Nacht zum Tag gemacht und Zeitinseln wie Sonntage und Festtage durch die Omnipräsenz elektronischer Medien aufgelöst. Es liegt heute hauptsächlich am Einzelnen, aus der ständigen Erreichbarkeit und der ruhelosen Tätigkeit auszubrechen, um aktive Zeiten mit Zeiten der Ruhe zu verbinden und das Auseinanderdriften von chronologisch-linearer Zeit und gelebter Zeit, von Weltzeit und Lebenszeit zu beschränken.

Ohne Ruhe ist der Mensch der Zeit ausgeliefert. Er vermag das Beständige nicht wahrzunehmen. Erst wenn dem Verweilen eine Chance gegeben wird, kann eine Gegenwärtigkeit erfahren werden, die zeitlos scheint, auch weil in ihr Vergangenheit und Zukunft mit enthalten sind.

Das neuste Buch des Autors «Krankheit als seelische Herausforderung» ist 2013 bei Schwabe erschienen. 